Article

\title{
A Reliable Method for Solving Fractional Sturm-Liouville Problems
}

\author{
M. M. Khashshan ${ }^{1}$, Muhammed I. Syam ${ }^{2, *}$ and Ahlam Al Mokhmari ${ }^{2}$ \\ 1 Department of Mathematics, Teachers College, King Saud University, Riyadh 4545, Saudi Arabia; \\ drmohammedr@yahoo.com \\ 2 Department of Mathematical Sciences, United Arab Emirates University, Al Ain 15551, UAE; \\ 201150059@uaeu.ac.ae \\ * Correspondence: m.syam@uaeu.ac.ae
}

Received: 18 August 2018; Accepted: 30 August 2018; Published: 26 September 2018

Abstract: In this paper, a reliable method for solving fractional Sturm-Liouville problem based on the operational matrix method is presented. Some of our numerical examples are presented.

Keywords: caputo derivative; operational matrix; fractional eigenvalue problem

\section{Introduction}

The Sturm-Liouville theory plays an important role for the development of spectral methods and the theory of self-adjoint operators [1]. Several applications on SLPs are studied as boundary-value problems [2]. The Sturm-Liouville eigenvalue problem has played an important role in modeling many physical problems. The theory of the problem is well developed and many results have been obtained concerning the eigenvalues and corresponding eigenfunctions. It should be noted that, since finding analytical solutions for this problem is an extremely difficult task, several numerical algorithms have been developed to seek approximate solutions. However, to date, mostly integer-order differential operators in SLPs have been used, and such operators do not include any fractional differential operators. Fractional calculus is a theory which unifies and generalizes the notions of integer-order differentiation and integration to any real order [3-5].

Recently, the fractional Sturm-Liouville problems were formulated in [6,7]. Authors in these papers considered several types of the fractional Sturm-Liouville equations and they investigated the eigenvalues and eigenfunctions properties of the fractional Sturm-Liouville operators.

Djrbashian [8] studied the existence of a solution to the fractional boundary value problem. In [9], authors discussed the aforementioned relation between eigenvalues and zeros of Mittag-Leffler function. In [10], they used the Homotopy Analysis method while, in [11], they used the fractional differential transform method to compute the eigenvalues. In [12], researchers used the Fourier series to solve this problem while, in $[13,14]$, they used the method of Haar wavelet operational matrix. In [15-19], researchers extended the scope of some spectral properties of fractional Sturm-Liouville problems. Variational methods and Inverse Laplace transform method were applied in [20,21], respectively. Recently, in [22], authors constructed numerical schemes using radial basis functions while, in [23], they used Galerkin finite element method for such problems. Greenberg and Marletta [24,25] developed their own code using Theta Matrices (SLEUTH). In [26], researchers implemented the iterated variation method.

In this article, we present a numerical technique for solving class of FSLPs of the form

$$
D^{\gamma}\left[f(t) D^{\gamma} y(t)\right]+\mu g(t) y(t)=h(t), 0 \leq t \leq 1, \frac{1}{2}<\gamma \leq 1
$$


subject to

$$
\begin{aligned}
& c_{0} y(0)+c_{1} D^{\gamma} y(0)=0, c_{0}^{2}+c_{1}^{2}>0 \\
& c_{2} y(1)+c_{3} D^{\gamma} y(1)=0, c_{2}^{2}+c_{3}^{2}>0
\end{aligned}
$$

where $c_{0}, c_{1}, c_{2}$ and $c_{3}$ are constants such that $\operatorname{det}\left(\begin{array}{ll}c_{0} & c_{1} \\ c_{2} & c_{3}\end{array}\right) \neq 0, f(t), g(t), h(t)$ are continuous functions with $f(t), g(t)>0$ for all $t \in[0,1]$, and $D^{\gamma}$ is the Caputo derivative.

Next, we present some results related to the Caputo fractional derivative, as well as the definition of the fractional-order functions.

Definition 1. The Rimann-Liouville fractional integral operator $I^{\gamma}$ of order $\gamma>0$ on $L_{1}[0,1]$ is given by

$$
\begin{aligned}
I^{\alpha} y(t) & =\frac{1}{\Gamma(\gamma)} \int_{0}^{t} \frac{y(s)}{(t-s)^{1-\gamma}} d s \\
I^{0} y(t) & =y(t)
\end{aligned}
$$

where $\Gamma(\gamma)$ is the Euler Gamma functionv (see $[5,27])$.

For any $\gamma, \zeta \geq 0$, and $\zeta>-1, I^{\alpha}$ existsfor any $t \in[0,1]$ and

$$
I^{\gamma} t^{\zeta}=\frac{\Gamma(\zeta+1)}{\Gamma(\zeta+\gamma+1)} t^{\zeta+\gamma}
$$

Definition 2. The Caputo fractional derivative of order $\gamma$ is defined by

$$
D^{\gamma} y(t)=I^{n-\gamma} D^{l} y(t)=\frac{1}{\Gamma(l-\gamma)} \int_{0}^{t} \frac{y^{(l)}(s)}{(t-s)^{\gamma-l+1}} d s,
$$

provided that the integral exists, where $l=[\gamma]+1,[\gamma]$ is the integer part of the positive real number $\gamma, t>0$.

For $y \in L_{1}[0,1]$ and $\gamma \geq 0$ :

$$
I^{\gamma} D^{\gamma} y(t)=y(t)-\sum_{r=0}^{l-1} y^{(r)}\left(0^{+}\right) \frac{t^{r}}{r !^{\prime}}
$$

Let $\Delta_{n}$ be defined by

$$
\Delta_{n}=\operatorname{Span}\left\{1, t^{\gamma}, t^{2 \gamma}, \ldots, t^{n \gamma}\right\} .
$$

The inner product on the set $\Delta_{n}$ is given by

$$
(f(t), g(t))={ }_{0}^{1} f(t) g(t) d t .
$$

Theorem 1. The sequence of functions defined as follows are orthogonal:

$$
y_{i}(t)=\left(t^{\gamma}-a_{i}\right) y_{i-1}(t)-b_{i} y_{i-2}(t), i=2,3, \ldots
$$

with $y_{0}(t)=1, y_{1}(t)=t^{\gamma}-a_{1}$, and

$$
a_{i}=\frac{\left(t^{\gamma} y_{i-1}(t), g(t)\right)}{\left(y_{i-1}(t), y_{i-1}(t)\right)}, b_{i}=\frac{\left(t^{\gamma} y_{i-1}(t), y_{i-2}(t)\right)}{\left(y_{i-2}(t), y_{i-2}(t)\right)}
$$


Proof. For $i=1$,

$$
\begin{aligned}
\left(y_{1}(t), y_{0}(t)\right) & =\left(t^{\gamma}-a_{1}, y_{0}(t)\right) \\
& =\left(t^{\alpha}, 1\right)-\frac{\left(t^{\alpha}, 1\right)}{(1,1)}(1,1)=0 .
\end{aligned}
$$

Assume the result of the theorem is true for $i>1$. Then, for any $j \in\{0,1, \ldots, i-2\}$, we have

$$
\begin{aligned}
\left(y_{i+1}(t), y_{j}(t)\right) & =\left(\left(t^{\gamma}-a_{i+1}\right) y_{i}(t)-b_{i+1} y_{i-1}(t), y_{j}(t)\right) \\
& =\left(t^{\gamma} y_{i}(t), y_{j}(t)\right)-a_{i+1}\left(y_{i}(t), y_{j}(t)\right)-b_{i+1}\left(y_{i-1}(t), y_{j}(t)\right) \\
& =\left(t^{\gamma} y_{i}(t), y_{j}(t)\right) \\
& =\left(y_{i}(t), t^{\gamma} y_{j}(t)\right) \\
& =\left(y_{i}(t), y_{j+1}(t)+a_{j+1} y_{j}(t)+b_{j+1} y_{j-1}(t)\right) \\
& =\left(y_{i}(t), y_{j+1}(t)\right)+a_{j+1}\left(y_{i}(t), y_{j}(t)\right)+b_{j+1}\left(y_{i}(t), y_{j-1}(t)\right) \\
& =0 .
\end{aligned}
$$

For $j=i-1$,

$$
\begin{aligned}
\left(y_{i+1}(t), y_{i-1}(t)\right) & =\left(\left(t^{\gamma}-a_{i+1}\right) y_{i}(t)-b_{i+1} y_{i-1}(t), y_{i-1}(t)\right) \\
& =\left(t^{\gamma} y_{i}(t), y_{i-1}(t)\right)-a_{i+1}\left(y_{i}(t), y_{i-1}(t)\right)-b_{i+1}\left(y_{i-1}(t), y_{i-1}(t)\right) \\
& =\left(t^{\gamma} y_{i}(t), y_{i-1}(t)\right)-b_{i+1}\left(y_{i-1}(t), y_{i-1}(t)\right) \\
& =\left(t^{\gamma} y_{i}(t), y_{i-1}(t)\right)-\frac{\left(t^{\gamma} y_{i}(t), y_{i-1}(t)\right)}{\left(y_{i-1}(t), y_{i-1}(t)\right)}\left(y_{i-1}(t), y_{i-1}(t)\right) \\
& =0
\end{aligned}
$$

\section{Operational Matrices of Fractional Integration}

A set of $l$ Block Pulse Functions (BPFs) in the interval $[0,1)$ are given by $\left\{b_{0}(t), b_{1}(t), \ldots, b_{l-1}(t)\right\}$ such that

$$
b_{i}(t)=\left\{\begin{array}{cc}
1, & \frac{i}{l} \leq t<\frac{i+1}{l} \\
0, & \text { otherwise }
\end{array}\right\}
$$

for $i=0,1, . ., l-1$. The following are some of the BPFs properties

$$
b_{i}(t) b_{j}(t)=\left\{\begin{array}{ll}
b_{i}(t), & i=j \\
0, & i \neq j
\end{array}\right\}
$$

and

$$
{ }_{0}^{1} b_{i}(t) b_{j}(t) d t=\left\{\begin{array}{ll}
\frac{1}{l}, & i=j \\
0, & i \neq j
\end{array}\right\} .
$$

If $y \in L_{2}[0,1]$, then

$$
y(t)=Y_{l-1}^{T} B_{l-1}(t)
$$

where

$$
Y_{l-1}=\left[\begin{array}{c}
y_{0} \\
y_{1} \\
\vdots \\
y_{l-1}
\end{array}\right], B_{l-1}(t)=\left[\begin{array}{c}
b_{0}(t) \\
b_{1}(t) \\
\vdots \\
b_{l-1}(t)
\end{array}\right]
$$


and

$$
y_{i}=l_{\frac{i}{l}}^{\frac{i+1}{l}} y(t) d t, i=0,1, \ldots l-1 .
$$

Theorem 2. Let I $I^{\gamma}$ be the Rimann-Liouville functional operator. Then,

$$
I^{\gamma} B_{l-1}(t)=P_{l}^{\gamma} B_{l-1}(t)
$$

where

$$
P_{l}^{\gamma}=\frac{1}{l \gamma} \frac{1}{\Gamma(\gamma+2)}\left[\begin{array}{ccccc}
1 & \varepsilon_{1} & \varepsilon_{2} & \ldots & \varepsilon_{l-1} \\
0 & 1 & \varepsilon_{1} . & \ldots & \varepsilon_{l-2} \\
0 & 0 & 1 & \ldots & \varepsilon_{l-3} \\
0 & 0 & 0 & \ddots & : \\
0 & 0 & 0 & 0 & 1
\end{array}\right]
$$

and $\varepsilon_{r}=(r+1)^{\gamma+1}-2 r^{\gamma+1}+(r-1)^{\gamma+1}, r=1: l-1$.

Proof. For each $i=0,1, \ldots, l-1$, we can write $I^{\gamma} b_{i}$ as

$$
I^{\gamma} b_{i}={ }_{j=0}^{l-1} c_{i j} b_{j}(t) .
$$

Multiply both sides by $b_{r}(t)$, for $0 \leq r \leq l-1$, then integrate both sides to get

$$
\begin{aligned}
c_{i r} & \left.=r_{l}^{\frac{r+1}{r}} I^{\gamma} b_{i}(t) d t=\frac{r}{\Gamma(\gamma)}\right)_{T}^{\frac{r+1}{l}} \int_{0}^{t} \frac{b_{i}(t)}{(t-t)^{1-\gamma}} d t d t . \\
& =\left\{\begin{array}{cc}
0, & i>r \geq 0 \\
1, & i=r \\
(r+1)^{\gamma+1}-2 r^{\gamma+1}+(r-1)^{\gamma+1} & i<r \leq l-1
\end{array}\right\} .
\end{aligned}
$$

For more details, see $[28,29]$.

Theorem 3. Let $Y_{M-1}(t)=\left[\begin{array}{c}y_{0}(t) \\ y_{1}(t) \\ \vdots \\ y_{M-1}(t)\end{array}\right]$. Then, there exists an $M \times l$ matrix $Q^{\gamma}$ such that

$$
Y_{M-1}(t)=Q_{M \times l}^{\gamma} B_{l-1}(t)
$$

where

$$
\left(Q_{M \times l}^{\gamma}\right)_{i, k}=l_{\frac{k}{l}}^{\frac{k+1}{l}} y_{i}(t) d t
$$

for $i=0: M-1$ and $k=0: l-1$.

Proof. It is easy to see that $y_{i}(t) \in L_{2}[0,1)$, for each $i=0: M-1$. Using Equations (10) and (11), we get

$$
Y_{M-1}(t)=Q_{M \times l}^{\gamma} B_{l-1}(t)
$$

where

$$
\left(Q_{M \times l}^{\gamma}\right)_{i, k}=l_{\frac{k}{l}}^{\frac{k+1}{l}} y_{i}(t) d t .
$$

for $i=0: M-1$ and $k=0: l-1$ which ends the proof.

From now on, let $M=l$. 
Theorem 4. If $0<\gamma<1$, then $Q_{l \times l}^{\gamma}$ is nonsingular matrix.

Proof. Theorem 3 implies that

$$
Y_{l-1}(t) Y_{l-1}(t)^{T}=Q_{l \times l}^{\gamma} B_{l-1}(t) B_{l-1}(t)^{T} Q_{l \times l}^{\gamma T}
$$

Integrate both sides with respect to $t$ on $(0,1)$ to get

$$
{ }_{0}^{1} Y_{l-1}(t) Y_{l-1}(t)^{T} d t=Q_{l \times l}^{\gamma}\left({ }_{0}^{1} B_{l-1}(t) B_{l-1}(t)^{T} d t\right) Q_{l \times l}^{\gamma T} .
$$

Theorem 1 and Equation (9) yield

$$
D_{1}=Q_{l \times l}^{\gamma} D_{2} Q_{l \times l}^{\gamma T}
$$

where

$$
D_{1}=\left[\begin{array}{cccc}
{ }_{0}^{1} y_{0}(t) y_{0}(t) d t & 0 & \cdots & 0 \\
0 & { }_{0}^{1} y_{1}(t) y_{1}(t) d t & \ddots & \vdots \\
\vdots & \ddots & \ddots & 0 \\
0 & \cdots & 0 & { }_{0}^{1} y_{l-1}(t) y_{l-1}(t) d t
\end{array}\right]
$$

and

$$
D_{2}=\frac{1}{\gamma}\left[\begin{array}{cccc}
\frac{1}{l \gamma} & 0 & \cdots & 0 \\
0 & \frac{2^{\gamma}-1}{l \gamma} & \ddots & \vdots \\
\vdots & \ddots & \ddots & 0 \\
0 & \cdots & 0 & \frac{l^{\gamma}-(l-1)^{\gamma}}{l \gamma}
\end{array}\right]
$$

Then, $\operatorname{det}\left(D_{1}\right)>0$ and $\operatorname{det}\left(D_{2}\right)>0$. Equation (14) gives

$$
\left(\operatorname{det}\left(Q_{l \times l}^{\gamma}\right)\right)^{2}=\frac{\operatorname{det}\left(D_{1}\right)}{\operatorname{det}\left(D_{2}\right)}>0
$$

Thus, $Q_{l \times l}^{\gamma}$ is nonsingular.

Operational Matrix of Fractional Integration

If $y \in C^{1}[0,1]$, then

$$
y(t)={ }_{k=0}^{\infty} u_{k} y_{k}(t)
$$

where

$$
u_{k}=\frac{{ }_{0}^{1} u(t) y_{k}(t) d t}{{ }_{0}^{1} f_{k}(t) y_{k}(t) d t} .
$$

Approximate the function $y(t)$ by

$$
U_{l-1}(t)={ }_{k=0}^{l-1} u y_{k}(t)=U^{T} Y_{l-1}(t),
$$

where

$$
U=\left[\begin{array}{c}
u_{0} \\
u_{1} \\
\vdots \\
u_{l-1}
\end{array}\right] \text { and } Y_{l-1}(t)=\left[\begin{array}{c}
y_{0}(t) \\
y_{1}(t) \\
\vdots \\
y_{l-1}(t)
\end{array}\right]
$$


Theorem 5. $I^{\gamma} Y_{l-1}(t)=H_{l}^{\gamma} Y_{l-1}(t)$ where

$$
H_{l}^{\gamma}=Q_{l \times l}^{\gamma} P_{l}^{\gamma}\left(Q_{l \times l}^{\gamma}\right)^{-1}
$$

Proof. Let $H_{l}^{\gamma}$ be given by

$$
I^{\gamma} Y_{l-1}(t)=H_{l}^{\gamma} Y_{l-1}(t)
$$

From Equations (13) and (17), we get

$$
I^{\gamma} Y_{l-1}(t)=H_{l}^{\gamma} Y_{l-1}(t)=H_{l}^{\gamma} Q_{l \times l}^{\gamma} B_{l-1}(t)
$$

and

$$
\begin{aligned}
I^{\gamma} Y_{l-1}(t) & =I^{\gamma} Q_{l \times l}^{\gamma} B_{l-1}(t) \\
& =Q_{l \times l}^{\gamma} I^{\gamma} B_{l-1}(t) \\
& =Q_{l \times l}^{\gamma} P_{l}^{\gamma} B_{l-1}(t) .
\end{aligned}
$$

Combining Equations (18) and (19), we get

$$
H_{l}^{\gamma} Q_{l \times l}^{\gamma} B_{l-1}(t)=Q_{l \times l}^{\gamma} P_{l}^{\gamma} B_{l-1}(t) .
$$

Therefore,

$$
H_{l}^{\gamma}=Q_{l \times l}^{\gamma} P_{l}^{\gamma}\left(Q_{l \times l}^{\gamma}\right)^{-1}
$$

\section{Method of Solution}

Using Equations (10) and (13), we get

$$
D^{\gamma}\left[f(t) D^{\gamma} y(t)\right]=U^{T} Y_{l-1}(t)=U^{T} Q_{l \times l}^{\gamma} B_{l-1}(t) .
$$

Thus,

$$
f(t) D^{\gamma} y(t)-f(0) \omega=I^{\gamma} U^{T} Y_{l-1}(t)
$$

where $\omega=D^{\gamma} y(0)$. Theorem 5 and Equations (10) and (13) imply that

$$
\begin{aligned}
D^{\gamma} y(t) & =\frac{1}{f(t)}\left(U^{T} I^{\gamma} Y_{l-1}(t)+f(0) \omega\right) \\
& =\frac{1}{f(t)}\left(U^{T} H_{l}^{\gamma} Y_{l-1}(t)+f(0) \omega\right) \\
& =U^{T} H_{l}^{\gamma} Q_{l \times l}^{\gamma} \frac{B_{m l-1}(t)}{f(t)}+\frac{f(0) \boldsymbol{\omega}}{f(t)} \\
& =U^{T} H_{l}^{\gamma} Q_{l \times l}^{\gamma}\left[\begin{array}{c}
b_{0}(t) / f(t) \\
b_{1}(t) / f(t) \\
\vdots \\
b_{l-1}(t) / f(t)
\end{array}\right]+f(0) \boldsymbol{\omega}\left[\begin{array}{c}
1 / f(t) \\
1 / f(t) \\
\vdots \\
1 / f(t)
\end{array}\right] .
\end{aligned}
$$

Hence,

$$
D^{\gamma} y(t)=\left(U^{T} H_{l}^{\gamma} Q_{l \times l}^{\gamma} P F_{1}+f(0) \omega F_{2}\right) B_{l-1}(t)
$$

Thus,

$$
y(t)=\left(U^{T} H_{l}^{\gamma} Q_{l \times l}^{\gamma} F_{1}+f(0) \omega F_{2}\right) I^{\gamma} B_{l-1}(t)+\psi
$$


where $\psi=y(0)$. Therefore,

$$
y(t)=\left(U^{T} H_{l}^{\gamma} Q_{l \times l}^{\gamma} F_{1}+f(0) \omega F_{2}\right) Y_{l}^{\gamma} B_{l-1}(t)+\psi .
$$

Hence,

$$
U^{T} Q_{l \times l}^{\gamma} B_{m-1}(t)+\mu g(t)\left(\left(U^{T} H_{l}^{\gamma} Q_{l \times l}^{\gamma} F_{1}+f(0) \omega F_{2}\right) F Y_{l}^{\gamma} B_{l-1}(t)+\psi\right)=h(t)
$$

or

$$
U^{T}\left(Q_{l \times l}^{\gamma}+\mu q(t) H_{l}^{\gamma} Q_{l \times l}^{\gamma} F_{1} Y_{l}^{\gamma}\right) B_{l-1}(t)=h(t)-\mu g(t) f(0) \omega F_{2} Y_{l}^{\gamma} B_{l-1}(t)-\mu \psi g(t) .
$$

Using the boundary conditions in Equations (2) and (3), we get the following cases

- if $c_{0}=0, \omega=0, c_{1} \neq 0, c_{2} \neq 0$, and

$$
\psi=-c_{2} U^{T} H_{l}^{\gamma} Q_{l \times m}^{\gamma} F_{1} Y_{l}^{\gamma} B_{l-1}(1)-\frac{c_{3}}{c_{2}} U^{T} H_{l}^{\gamma} Q_{l \times l}^{\gamma} P F_{1} B_{l-1}(1)
$$

- if $c_{0} \neq 0, \psi=-\frac{c_{1}}{c_{0}} \omega$ and

$$
\mathfrak{\omega}=\frac{-c_{2} U^{T} H_{l}^{\gamma} Q_{l \times l}^{\gamma} F_{1} Y_{l}^{\gamma} B_{l-1}(1)-c_{3} U^{T} H_{l}^{\gamma} Q_{l \times l}^{\gamma} Y_{1} B_{l-1}(1)}{f(0) F_{2} Y_{l}^{\gamma} B_{l-1}(1)-\frac{c_{1} c_{2}}{c_{0}}+c_{3} f(0) Y_{2} B_{l-1}(1)} .
$$

Thus,

$$
U^{T}\left(\begin{array}{c}
Q_{l \times l}^{\gamma}+\mu g(t) H_{l}^{\gamma} Q_{l \times l}^{\gamma} F_{1} Y_{l}^{\gamma} B_{l-1}(t)+ \\
\left(\mu g(t) f(0) F_{2} Y_{l}^{\gamma} B_{l-1}(t)-\frac{c_{1}}{c_{0}} \mu g(t)\right)\left(\frac{-c_{2} H_{l}^{\gamma} Q_{l \times l}^{\gamma} F_{1} Y_{l}^{\gamma} B_{l-1}(1)-c_{3} H_{l}^{\gamma} Q_{l \times l}^{\gamma} F_{1} B_{l-1}(1)}{f(0) F_{2} F_{l}^{\gamma} B_{l-1}(1)-\frac{c_{1} c_{2}}{c_{0}}+c_{3} f(0) F_{2} B_{l-1}(1)}\right)
\end{array}\right)=h(t) .
$$

We use the collocation points

$$
t_{i}=\frac{r+1}{l+1}, i=0: l-1 .
$$

Substitute these values into Equation (23) and take the transpose of both sides to get a system of linear equations in terms of $U$ of the form

$$
G(\mu) U=R .
$$

To have a nonzero solution to the system in Equation (24), $G(\mu)$ must be nonsingular. Thus,

$$
\operatorname{det}(G(\mu))=0 .
$$

Therefore, we find the eigenvalues from Equation (25) and we find the corresponding eigenfunctions from Equation (21).

\section{Numerical Results}

We present two examples for $l=16$. In this paper, we focus only on the eigenvalues.

Example 1. Consider

$$
\begin{gathered}
D^{\gamma}\left[D^{\gamma} y(t)\right]+\mu y(t)=0, t \in[0,1], \gamma \in(0.5,1], \\
y(0)=0, y(1)=0 .
\end{gathered}
$$


Using the procedure described in the previous section, the generated eigenvalues are reported in Table 1.

Table 1. Eigenvalues for different values of $\gamma$.

\begin{tabular}{ccc}
\hline $\boldsymbol{\gamma}=\mathbf{0 . 7 5}$ & $\boldsymbol{\gamma}=\mathbf{0 . 9 5}$ & $\boldsymbol{\gamma}=\mathbf{0 . 9 9}$ \\
\hline 8.7825905605957 & 8.2711826449023 & 9.6635258705797 \\
14.0844503539395 & 58.990163159836 & 38.044080578817 \\
& 96.673652759078 & 84.971438095925 \\
& 148.295350243613 & 150.13372170205 \\
& 199.571402506686 & 233.59863572826 \\
& 277.107135647923 & 335.09777723091 \\
& 295.450149615306 & 454.76440655382 \\
& & 590.93089519416 \\
\hline
\end{tabular}

For $\gamma=1$, the exact eigenvalues are well-known and they are given by

$$
\mu_{n}=n^{2} \pi^{2}, n=1,2,3, \ldots
$$

It is worth mentioning that the eigenvalues of the problem in this example approach to $n^{2} \pi^{2}$ when $\gamma$ approaches to 1 . Let

$$
\delta_{i, j}=\left|{ }_{0}^{1} y_{i}(t) y_{j}(t) d t\right|
$$

For $\gamma=0.75, \delta_{1,2}=5.7 \times 10^{-16}$. Sample of these values for $\gamma=0.95$ are given as

$$
\delta_{1,2}=5.7 \times 10^{-16}, \delta_{4,6}=2.6 \times 10^{-16}, \delta_{1,6}=8.3 \times 10^{-16} .
$$

Similarly, for $\gamma=0.99$,

$$
\delta_{1,2}=3.1 \times 10^{-16}, \delta_{4,6}=4.2 \times 10^{-16}, \delta_{1,7}=2.0 \times 10^{-16} .
$$

This means the orthogonality relation holds. We notice that the eigenvalues satisfy the increasing property.

Example 2. Consider

$$
\begin{gathered}
D^{\alpha}\left[D^{\alpha} y(t)\right]+\lambda\left(1+t^{\alpha}\right) y(t)=0, t \in[0,1], \gamma \in(0.5,1], \\
u(0)=0, u(1)=0 .
\end{gathered}
$$

Using the procedure described in the previous section, the generated eigenvalues are reported in Table 2.

Table 2. Eigenvalues for different values of $\gamma$.

\begin{tabular}{ccc}
\hline$\gamma=\mathbf{0 . 5 0 1}$ & $\gamma=\mathbf{0 . 7 5}$ & $\gamma=\mathbf{0 . 9 5}$ \\
\hline 3.74496847027704 & 4.90596508821842 & 5.82711926402061 \\
5.59359607314814 & 9.95423834570763 & 21.8630977993855 \\
25.47511927569108 & 14.2468657217155 & 100.868795211963 \\
151.8458499360075 & 25.8797084072818 & 234.225682145921 \\
& 124.475138197374 & 439.200912754629 \\
& & 721.009344587213 \\
& & 984.124781340994 \\
\hline
\end{tabular}


Let

$$
\delta_{i, j}=\left|{ }_{0}^{1} y_{i}(t) y_{j}(t) g(t) d t\right| .
$$

For $\gamma=0.502, \delta_{1,2}=3.3 \times 10^{-16}$ and $\delta_{2,4}=4.9 \times 10^{-16}$. Samples of these values for $\gamma=0.75$ are given as

$$
\delta_{1,2}=2.2 \times 10^{-16}, \delta_{4,5}=4.1 \times 10^{-16}, \delta_{1,5}=6.9 \times 10^{-16} .
$$

Similarly, for $\gamma=0.95$,

$$
\delta_{1,2}=1.2 \times 10^{-16}, \delta_{4,6}=2.1 \times 10^{-16}, \delta_{1,7}=4.6 \times 10^{-16} .
$$

This means the orthogonality relation holds. We notice that the eigenvalues satisfy the property

$$
\mu_{1} \leq \mu_{2} \leq \ldots
$$

\section{Conclusions}

In this article, a reliable method for solving fractional Sturm-Liouville problem based on the operational matrix method is presented. Two of our numerical examples are presented. From the previous discussion, we notice the following.

- $\quad$ From previous section, we can find the eigenvalues with the following property

$$
\lambda_{1}<\lambda_{2}<\lambda_{3}<\ldots<\lambda_{n}<\ldots
$$

- From previous section, the orthogonality property

$$
{ }_{0}^{1} y_{i}(t) y_{j}(t) q(t)=0, i \neq j
$$

holds.

- The proposed method can be generalized to other applications in Physics and Engineering.

Author Contributions: All authors have the same contributions.

Funding: This research received no external funding.

Conflicts of Interest: The authors declare no conflict of interest.

\section{References}

1. Amrein, W.O.; Hinz, A.M.; Pearson, D.B. Sturm-Liouville Theory: Pastand Present; Birkhäuser: Basel, Switzerland, 2005.

2. Zettl, A. Sturm-Liouville Theory; American Mathematical Society: Providence, RI, USA, 2010; Volume 121.

3. Miller, K.S.; Ross, B. An Introduction to the Fractional Calculus and Fractional Differential Equations; John Wiley and Sons, Inc.: New York, NY, USA, 1993.

4. Podlubny, I. Fractional Differential Equations; Academic Press: San Diego, CA, USA, 1999.

5. Kilbass, A.A.; Srivastava, H.M.; Trujillo, J.J. Theory and Applications of Fractional Differential Equations; Elsevier: Amsterdam, The Netherlands, 2006.

6. Klimek, M.; Agrawal, O.P. Fractional Sturm Liouville problem. Comput. Math. Appl. 2013, 66, 795-812. [CrossRef]

7. Rivero, M.; Trujillo, J.J.; Velasco, M.P. A fractional approach to the Sturm-Liouville problem. Cent. Eur. J. Phys. 2013, 11, 1246-1254. 10.2478/s11534-013-0216-2. [CrossRef]

8. Djrbashian, M.M. A boundary value problem for a Sturm-Liouville type differential operator of fractional order. Izv. Akad. Nauk Armjan. SSR Ser. Mat. 1970, 5, 71-96.

9. Nahusev, A.M. The Sturm-Liouville problem for a second order ordinary differential equation with fractional derivatives in the lower terms. Dokl. Akad. Nauk SSSR 1977, 234, 308-311. 
10. Syam, M.I.; Siyyam, H.I. An efficient technique for finding the eigenvalues of fourth-order Sturm-Liouville problems. Chaos Solitons Fractals 2009, 39, 659-665. [CrossRef]

11. Khan, J.A.; Raja, M.A.Z.; Syam, M.I.; Tanoli, S.A.K.; Awan, S.E. Design and application of nature inspired computing approach for nonlinear stiff oscillatory problems. Neural Comput. Appl. 2015, 26, 1763-1780. [CrossRef]

12. El-Sayed, M.F.; Syam, M.I. Electrohydrodynamic instability of a dielectric compressible liquid sheet streaming into an ambient stationary compressible gas. Arch. Appl. Mech. 2007, 77, 613-626. [CrossRef]

13. Attili, B.S.; Furati, K.; Syam, M.I. An efficient implicit Runge-Kutta method for second order systems. Appl. Math. Comput. 2006, 178, 229-238. [CrossRef]

14. Syam, M.I.; Siyyam, H.I. Numerical differentiation of implicitly defined curves. J. Comput. Appl. Math. 1999, 108, 131-144. [CrossRef]

15. Raja, M.A.Z.; Shah, F.H.; Alaidarous, E.S.; Syam, M.I. Design of bio-inspired heuristic technique integrated with interior-point algorithm to analyze the dynamics of heartbeat model. Appl. Soft Comput. J. 2017, 52, 605-629. [CrossRef]

16. Attili, B.S.; Syam, M.I. Efficient shooting method for solving two point boundary value problems. Chaos Soliton Fractals 2008, 35, 895-903. [CrossRef]

17. Syam, M.I. The modified Broyden-variational method for solving nonlinear elliptic differential equations, Journal of Function Spaces and Applications. Chaos Soliton Fractals 2007, 32, 392-404. [CrossRef]

18. Syam, M.I.; Attili, B.S. Numerical solution of singularly perturbed fifth order two point boundary value problem. Appl. Math. Comput. 2005, 170, 1085-1094. [CrossRef]

19. Aboelenen, T.; El-Hawary, H.M. Spectral Theory and Numerical Approximation for Singular Fractional Sturm-Liouville eigen-Problems on Unbounded Domain. arXiv 2014, arXiv:1410.1583v4.

20. Klimek, M.; Odzijewicz, T.; Malinowska, A.B. Variational Methods for the Fractional Sturm-Liouville Problem. J. Math. Anal. Appl. 2014, 416, 402-426. [CrossRef]

21. Saei, F.D.; Abbasi, S.; Mirzayi, Z. Inverse Laplace transform method for multiple solutions of the fractional Sturm-Liouville problems. Comput. Methods Differ. Equ. 2014, 2, 56-61.

22. Antunes, P.; Ferreira, R. An augmented-RBE method for solving fractional Sturm-Liouville eigenvalues problems. SIAM J. Sci. Comput. 2015, 37, A515-A535. [CrossRef]

23. Jin, B.; Lazarov, R.; Lu, X.; Zhou, Z. A simple finite element method for boundary value problems with a Riemann-Liouville derivative, Journal of Computational and Applied Mathematics. arXiv 2015, arXiv:1502.07840v1.

24. Greenberg, L. An oscillation method for fourth order self-adjoint two-point boundary value problems with nonlinear eigenvalues. SIAM J. Math. Anal. 1991, 22, 1021-1042. [CrossRef]

25. Greenberg, L.; Marletta, M. Algorithm 775: The code SLEUTH for solving fourth order Sturm-Liouville problems. ACM Trans. Math. Softw. 1997, 23, 453-493. [CrossRef]

26. Syam, M.; Siyyam, H. An Efficient Technique for Finding the Eigenvalues of Sixth-Order Sturm-Liouville problems. Appl. Math. Sci. 2011, 5, 2425-2436.

27. Caputo, M. Linear models of dissipation whose $\mathrm{Q}$ is almost frequency independent, Part II. Geophys. J. Int. 1967, 13, 529-539. [CrossRef]

28. Li, Y.; Sun, N. Numerical solution of fractional differential equations using the generalized block pulse operational matrix. Comput. Math. Appl. 2011, 62, 1046-1054. [CrossRef]

29. Pandey, R.; Bhaedwaj, A.; Syam, M.I. An efficient method for solving fractional differential equations using Bernstein polynomials. J. Fract. Calc. Appl. 2014, 5, 129-145.

(c) 2018 by the authors. Licensee MDPI, Basel, Switzerland. This article is an open access article distributed under the terms and conditions of the Creative Commons Attribution (CC BY) license (http://creativecommons.org/licenses/by/4.0/). 